\title{
Indian Academy of Pediatrics Revised Guidelines on School Reopening: First Revision, September 2021
}

\author{
Swati Ghate, ${ }^{1}$ Siddhesh Zadey, ${ }^{2}$ Rajeev Kumar Thapar, ${ }^{3}$ Dheeraj Shah, ${ }^{4}$ GV Basavaraja, ${ }^{5}$ SS Kamath,${ }^{6}$ \\ Ramesh Kumar R, ${ }^{7}$ Nimesh G Desai,${ }^{8}$ Bakul Jayant Parekh,,${ }^{9}$ Vipin M Vashishtha, ${ }^{10}$ AshoK Pandey, ${ }^{11}$ \\ PIYUSH GUPTA ${ }^{12}$ \\ ${ }^{1}$ Babylon's Newton Child development and Support Centre, Jaipur, Rajasthan, ${ }^{2}$ Duke UniversitySchool of Medicine, Durham, NC, \\ USA, ${ }^{3}$ School of Medicine \& Research, Sharda Hospital, Greater Noida, UP; ${ }^{4}$ Departments of Pediatrics, University College of \\ Medical Sciences and GTB Hospital, Delhi; ${ }^{5}$ Indira Gandhi Children's Hospital, Bengluru, Karnataka, ${ }^{6}$ Indira Gandhi Cooperative \\ Hospital, Kochi; Kerala $;{ }^{7}$ Apollo Adlux Hospital, Kochi, Kerala, ${ }^{8}$ Institute of Human Behaviour and Allied Sciences, Delhi, ${ }^{9}$ Bakul \\ Parekh Hospital for Children, Mumbai, Maharashtra; ${ }^{10}$ Mangla Hospital, Bijnor, ${ }^{11}$ Ahlcon Public and International Schools, \\ Delhi, ${ }^{12}$ President, Indian Academy of Pediatrics, Mumbai, Maharashtra. \\ Correspondence to: Dr Piyush Gupta, Professor and Head, Department of Pediatrics, University College of Medical Sciences and \\ GTB Hospital,Delhi.prof.piyush.gupta@gmail.com
}

Justification:The COVID-19 pandemic has affected schooling for more than 24 crores students, since March 2020. Students need a respite from the long standing social isolation so that they regain their chance to develop holistically, but after the devastating effects of the second wave, the administrators as well as parents are skeptical about the decision of school reopening. Process: The Indian Academy of Pediatrics constituted a task force comprising of national and international experts in the field who deliberated on the issue. Objectives: To bring out scientifically supported guidelines on the prerequisites of opening and attending the schools, in the current context of the COVID-19 pandemic. Recommendations:The task force recommends $i$ ) Decentralization of the school reopening decision; ii)Three epidemiological parameters, case positivity rate ( $<5$ or steadily declining number of cases for past two weeks), number of new cases ( $<20$ per lakh population per day for past two weeks) and vaccination coverage ( $>60 \%$ of the vaccine-eligible population) to be met at the local level, before the schools reopen; and iii) Criteria regarding health and vaccination to be met by the schoolattendees.

Keywords: COVID-19, Education, Formal education, Pandemic.

$\mathrm{D}$ uring the COVID-19 pandemic, crores of children and adolescents are negatively affected across various domains of their health and development [1]. With prolonged school closures, the educational void is increasing day by day and education is probably suffering the most [2]. An Indian survey study on more than 16000 students across five states has shown that, about $92 \%$ and $82 \%$ children have declined in (at least one) mathematical and linguistic abilities, respectively [3]. Another survey study conducted across 15 States revealed the gross failure of online learning methodology to reach out to all the students. Only $24 \%$ students from urban area and $8 \%$ from rural area are reported to study online regularly; $37 \%$ rural children are completely out of the education stream [4]. Children are homebound for more than 17 months now. Consequently, they are displaying significant physical and psychosocial health issues [1]. Reopening of the schools and bringing back normalcy in their lives is eagerly awaited.

It has been amply proved that more than half of the children infected with COVID -19 are either asymptomatic or develop mild form of the disease [5]. There are studies to prove that schools do not act as major sources of viral transmission [6]. Outbreaks have been reported where the mitigation measures were not strictly adhered to [7].

The latest body of research on COVID-19, particularly in children, and the advent and the availability of the vaccines, are scientifically favorable factors for relaxing the social restrictions imposed on children. But the horrific experiences during the second wave are unforgettable and act as a big barrier when there is any attempt to bring the children back to schools. The Government authorities, the school administrators, as well as the parents appear to be in a state of dilemma and confusion. The decision for school reopening therefore, needs to be taken meti-culously and with scientific evidences.

To address the issues of schooling during the pandemic, the Indian Academy of Pediatrics (IAP) had framed recommendations on 'School Reopening, Remote Learning and Curriculum during and after COVID-19 Pandemic', in October, 2020 [8]. We, herein, provide an update to these guidelines. 


\section{OBJECTIVES}

To frame guidelines to readdress the epidemiological parameters for school reopening, and to formulate recommendations for individuals (staff, students and visitors) while attending the school.

\section{METHODOLOGY}

The Indian Academy of Pediatrics constituted an expert panel, the 'Task Force on School Reopening 2021' in August, 2021. The panel members exchanged their ideas and thoughts through Zoom meetings, group mails and other social networks. They studied relevant latest research papers and articles, other currently released guidelines and took into consideration, the experiences of school reopening at various places across the globe. After deliberating on the matter, the guidelines were drafted and circulated to all the members for their suggestions and approval. The Task Force thereafter finalized the following revised recommendations on 'when' to open the schools:

\section{RECOMMENDATIONS}

\section{General Recommendations}

- Decision-making regarding the school reopening should be decentralized. It should be taken at the level of districts (or taluka/city/village/school) according to the local situations, rather than at the national or state level.

- Strict compliance to the COVID appropriate behaviour (social distancing/masking/sanitization/respiratory hygiene etc.) by each and everyone in the community should be encouraged and ensured.

- Isolation, testing and contact tracing of symptomatic school attendees should be carried out as per local health guidelines.

- Healthcare system should be adequately geared up to handle any potential outbreaks through meticulous microplanning.

- Local COVID-19 statistics should be vigilantly followed. The decision regarding keeping the schools open should be reviewed every 15 days.

- COVID vaccination drives should be undertaken to cover vaccine eligible population as early as possible.

\section{Criteria for School Reopening}

These criteria need to be met at the local level.

1. The case positivity rate for COVID-19 (i.e., the number of RT-PCR positive per hundred tests) should be less than $5 \%$ for the preceding two weeks OR if the case positivity rate is greater than $5 \%$, the total number of new cases should have been steadily declining over the preceding two weeks [8].
2. Number of new cases per 1 lakh population per day should be less than 20 for preceding two weeks [8].

3. Vaccination coverage (as measured by at least one dose of any of the COVID vaccines) of the adult (or the vaccine-eligible) population should be $60 \%$ or more $[9,10]$.

When all these three criteria are met, the local authorities may consider opening of the schools.

\section{Criteria for Attending the School}

- All the adult members working at and closely associated with schools, including the teachers, nonteaching staff, support staff, commute drivers, attendants, and school visitors, should have received at least one dose of any COVID-19 vaccine.

- Students above the age of 18 years should have received at least one dose of COVID-19 vaccine.

- Both parents and all other household adults of the student should have received at least one dose of COVID-19 vaccine.

- Parents should be willing and should give consent for sending the ward to the school.

- High risk staff members (like those suffering from diabetes, high blood pressure, obesity, lung diseases etc.) should have completed two doses of COVID -19 vaccination 15 days prior to joining the schools.

- High-risk children (like those suffering from chronic diseases like asthma, kidney diseases, children with special needs, children on steroids etc.) should consult their pediatricians before joining the schools.

- Anyone who is not feeling well should refrain from attending the school and consult a medical professional before returning to the school.

The current evidence suggests that the seroprevalence is a highly volatile indicator with high false positivity and it is not a robust criterion for policy decisions on social restrictions. The results of seroprevalence surveys are influenced by several factors like restricted populations, non-representative samples, uncertainty around test accuracy, and limited knowledge about presence of antibodies in the long term. Further, these are required to be interpreted in the context of other external information, such as confirmed cases, deaths, and infectious disease models, to understand the disease better[11,12].

IAP will update the advisory as and when new scientific data emerges.

Funding: None; Competing interests: None stated. 
Contributors: All authors were part of the IAP Task Force on School Reopening, 2021, that formulated these guidelines. PG, GVB, BJP and SG conceived the guidelines, prepared the agenda, and executed administratively. PG led the discussions and all the members actively participated. DS, RR, SSK, SZ, GVB contributed on the epidemiological aspects. ND and VMV contributed on the vaccination issues. SG and SZ reviewed the literature. SG and SZ wrote the first draft. ND and AP suggested edits. RKT and PG did the final edits. All the authors approved the final recommendations of the guidelines.

\section{REFERENCES}

1. Unicef Report. Press release, March 2021. Accessed on 4 September 2021.Available from: https://www.unicef.org/press-releases/schools-more-168-million-children-globally-havebeen-completely-closed

2. Araújo LA, Veloso CF, Souza MC, Azevedo JMC, Tarro G. The potential impact of the COVID-19 pandemic on child growth and development: a systematic review. J Pediatr (Rio J). 2021;97:369-77.

3. Azim Premji Foundation Research Group. Loss of Learning during the Pandemic. Azim Premji University; February 2021.Accessed on 13 Sep, 2021. Available from:https://archive azimpremjiuniversity. edu.in/SitePages/pdf/Field_Studies_Loss_of_Learning_during_the Pandemic.pdf

4. The SCHOOL Team. Locked out: Emergency Report on School Education. September 2021.Accessed on 13 Sep, 2021. Available from:https://www.google.com/url? sa $=t \&$ source $=w e b \& r c t=j \& u r l=$ https://counterviewfiles.files.wordpress.com/2021/09/lockedout-emergency-report-on-school-education-6-sept-2021

5. Cui X, Zhao Z, Zhang T, et al. A systematic review and metaanalysis of children with coronavirus disease 2019 (COVID-19). J Med Virol. 2021;93:1057-69.

6. UNICEF. In-Person Schooling and COVID-19 Transmission: A Review of The Evidence; 2020. Accessed on 4 Sep, 2021. Available from: www.unicef.org/documents/in-person-schooling-covid19-transmission-review-of-evidence

7. Stein-Zamir C, Abramson N, Shoob H, et al. A large COVID-19 outbreak in a high school 10 days after schools' reopening, Israel, May 2020. Euro Surveill. 2020;25:2001352.

8. Ghate S, Parekh BJ, Thapar RK, et al. Indian Academy of Pediatrics Guidelines on School Reopening, Remote Learning and Curriculum in and After the COVID-19 Pandemic. Indian Pediatr. 2020; 57:1153-165.

9. Wang W, Wu Q, Yang J, et al. Global, regional, and national estimates of target population sizes for covid-19 vaccination: descriptive study. BMJ. 2020 Dec 15;371:m4704.

10. World Health Organization. Coronavirus Disease (covid-19) Herd Immunity. WHO; 2020. Accessed on 4 September, 2021. https://www.who.int/emergencies/diseases/novel-coronavirus2019/mediaresources/science-in-5/episode-1

11. Brownstein NC, Chen YA. Predictive values, uncertainty, and interpretation of serology tests for the novel coronavirus. Sci Rep. 2021;11:5491.

12. McConnell D, Hickey C, Bargary N, et al. Understanding the challenges and uncertainties of seroprevalence studies for SARS-CoV2. Int J Environ Res Public Health. 2021;18:4640. 